\title{
On Drawing a Graph Convexly in the Plane (Extended Abstract) *
}

\author{
HRISTO N. DJIDJEV \\ Department of Computer Science, Rice University, Hoston, TX 77251, USA
}

\begin{abstract}
Let $G$ be a planar graph and $H$ be a subgraph of $G$. Given any convex drawing of $H$, we investigate the problem of how to extend the drawing of $H$ to a convex drawing of $G$. We obtain a necessary and sufficient condition for the existence and a linear algorithm for the construction of such an extension. Our results and their corollaries generalize previous theoretical and algorithmic results of Tutte, Thomassen, Chiba, Yamanouchi, and Nishizeki.
\end{abstract}

\section{Introduction}

The problem of embedding of a graph in the plane so that the resulting drawing has nice geometric properties has received recently significant attention. This is due to the large number of applications including circuit and VLSI design, algorithm animation, information systems design and analysis. The reader is referred to [1] for annotated bibliography on graph drawings.

The first linear-time algorithm for testing a graph for planarity was constructed by Hopcroft and Tarjan [9]. A different linear time algorithm was developed by Booth and Lueker [3], who made use of $P Q$-trees and a previous algorithm of Lempel et al. [11]. Using the information obtained during the planarity testing, one can find in linear time a planar representation of the graph, if it is planar. Such an algorithm based on [3] has been described by Chiba et al. [4].

A classical result established by Wagner states that every planar graph has a planar straight-line drawing [18]. Since Wagner's result a lot of researchers have investigated various problems of finding straight-line drawings satisfying additional requirements, e.g., drawing the vertices of the graph on the points of a grid of small size, maximizing the minimum angle between adjacent segments of the drawing, upward straight-line drawings [1]. One of the oldest results in this field concerns straight-line drawings with the constraint that all the faces be convex polygons. Such drawings are called convex drawings. Stein [14] showed that a convex drawing always exists if the common boundary between any two faces is connected. Tutte [16] proved that every triconnected graph has a convex drawing and such a drawing can be constructed by solving a system of linear equations [17]. Linear algorithms that find grid convex drawings with quadratic area for triconnected graphs were given by Kant [10], Schnyder and Trotter [13], and Chrobak and Kant [6]. Di Battista et al. [2] consider the on-line version of the convex drawing problem.

Thomassen [15] gives a necessary and sufficient condition for existence of a convex drawing of an arbitrary (i.e. not necessarily triconnected) planar graph, if a convex drawing of the outer face has been previously fixed. Based on the Thomassen's proof,

* This work is partially, supported by National Scientific Foundation grant CCR-9409191. 

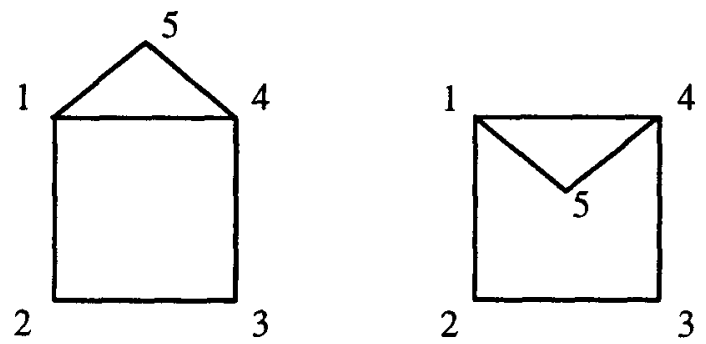

Fig. 1. A convex and a non-convex planar drawing.

Chiba et al. [5] give a linear-time algorithm to construct such an extension if one exists. The choice of the outer face can, however, be crucial for the existence of a planar drawing. For example, the graph on Figure 1 has a convex drawing in the plane if the face 1-2-34-5-1 is chosen to be the outer face, but has no convex drawing with outer face 1-2-3-4-1. Chiba et al. [5] reduce the problem of finding a suitable outer face (if one exists) to a planarity testing of a graph defined by the set of separation pairs of $G$.

This paper contains some new theoretical and algorithmic results associated with the problem of drawing a planar graph convexly. More specifically, we investigate the problem of extending a partially drawn graph in the plane to a convex drawing of the whole graph. We solve this problem by giving a necessary and sufficient condition for a convex drawing of a subgraph $H$ of a planar graph $G$ to be extendible to a convex drawing of $G$. This generalizes the previous results of Tutte and Thomassen who restrict $H$ to be an outer facial cycle. We show how to convert our proofs into linear-time algorithms for constructing the corresponding convex extensions. Finally, we address the problem of convex testing: determine if a planar graph has a convex embedding and construct one if the answer is yes. Here we have no restrictions on the embedding (e.g. we have the freedom of choosing any cycle to be the outer face). We show that this problem can be solved using our main result for extending convex drawings. Our algorithm has the same asymptotic bound as the optimal algorithm due to Chiba et al., but ours is significantly simpler.

The paper is organized as follows. Section 2 contains simple facts and definitions of terms used in the paper. Section 3 contains the formulation and the proof of our main theorem as well as some of its corollaries. Algorithmic problems associated with convex testing and convex drawing are discussed in Section 4.

\section{Preliminaries}

A topologacal embedding $\mu=\mu(G)$ of a graph $G$ in the plane is a mapping of $G$ onto the plane such that vertices are embedded onto distinct points and edges are embedded onto simple curves and no two different edges intersect except possibly at a common endpoint. A graph $G$ is planar if there exists a topological embedding of $G$ in the plane. The faces of the embedding are the boundaries of the connected regions obtained by deleting the embedding of $G$ from the plane. The unbounded face is called outer face of $\mu(G)$ and will be denoted by $O F(\mu(G))$ (or simply $O F(G)$, if the drawing $\mu(G)$ is clear from the context).

The topological definition of a graph embedding given above is not very convenient for computer representation of graph embeddings and for the analysis of algorithms. 
Each topological embedding induces a combınatorial embedding which is defined as an assignment of a cycling ordering to the set of edges incident to each vertex. This ordering of the edges corresponds to the order in which the edges are embedded around each vertex clockwise. The planarity testing algorithms from [9] and [3] produce only combinatorial embeddings. To each combinatorial embedding of $G$ an infinite number of topological embeddings of $G$ correspond. In the context of this paper we will call any topological embedding of $G$ a drawing of $G$.

A convex drawing $\mu(G)$ of a planar graph $G$ is a drawing of $G$ in the plane such that each face of $C$ is a convex polygon and the outer face is a strictly convex polygon. If the drawing of a specific face of $G$ is strictly convex, the drawing of the face is called a strictly convex drawing. Consider some (possibly non-convex) drawing $\mu(G)$ of $G$ in the plane such that the drawing $\mu(H)$ of a specific subgraph $H$ of $G$ is convex. By convexly extending $\mu(H)$ to $\mu(G)$ we will mean defining a new drawing $\mu^{\prime}(G)$ of $G$ in the plane such that $\mu^{\prime}(G)$ is a convex drawing of $G, \mu^{\prime}(G)$ contains the drawing $\mu(H)$ of $H$, and $\mu(G)$ and $\mu^{\prime}(G)$ correspond to the same combinatorial embedding of $G$. An embedding $e m b(H)$ of $H$ is convexly extenduble to $G$, if there exists a convex drawing of $G$ whose combinatorial embedding induces $e m b(H)$.

If $C^{\prime}$ and $G^{\prime}$ are any graphs, by $G+G^{\prime}$ we will denote the graph with vertex set $V\left(C_{r}\right) \cup V\left(G^{\prime}\right)$ and edge set $E(G) \cup E\left(G^{\prime}\right)$. Moreover, by $G_{r}-G^{\prime}$ we will denote the subgraph of $G^{*}$ induced by the set of vertices $V(G)-V\left(G^{\prime}\right)$. If $V^{*}$ is any set of vertices of $G, G-V^{*}$ will denote the subgraph of $G$ induced by the set of vertices $V(G)-V^{*}$.

Let $H$ be a subgraph of $G$. A bridge of $G-H$ is any subgraphs $B$ of $G$ induced by a maximal set of edges such that any two different edges of $B$ can be joined by a path containing no vertices of $H$. The edges of $B$ with an endpoint in $H$ are called attachment edges. We call $H$ a chordless subgraph, if any edge joining two vertices of $H$ belongs to $H$ and we call $H$ outer-chordless with respect to a given planar drawing of $G$, if no edge joining two vertices of $H$ is drawn in the outer face of $H$. A cycle $c^{\prime}$ is called a reduction of a cycle $c$ (respectively an outer reduction of $c$ ) if all vertices of $c^{\prime}$ belong to $c$ and $c^{\prime}$ is chordless (respectively outer-chordless) cycle.

For the standard graph theoretic definitions the reader is referred to any of the textbooks on graph theory.

\section{Extending a Convex Drawing}

In this section we obtain a necessary and sufficient condition for the existence of a convex extension of a planar drawing.

Theorem 1 Let $G$ be a planar biconnected graph and $H$ be a biconnected subgraph of $G$. Let $\mu(G)$ be a planar drawing of $G$ such that the induced drawing $\mu(H)$ of $H$ is convex and $O F(G)$ contains $O F(H)$. Then $\mu(H)$ can be convexly extended to $\mu(G)$ if and only if the following four conditions are satisfied (see Figure 2):

(a) For each vertex $v$ of $G-O F(G)$ of degree at least three in $G$, there exist three paths joining $v$ to vertices of $O F(G)$ such that no two paths share a common vertex except $v$.

(b) Any cycle of $G$ with no edge in common with $O F(G)$ has at least three vertaces of degree greater than two in $(\mathrm{H}$.

(c) Any connected component of $G-H$ adjacent only to vertuces belonging to the same straight-line of $\mu(H)$ is drawn outside $O F(H)$ and is adjacent to two vertices of degree greater than two. 


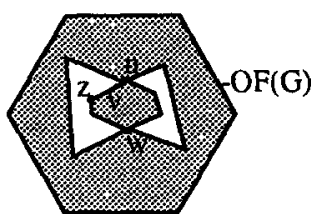

(a)

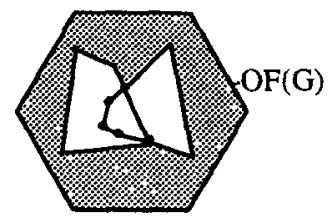

(b)

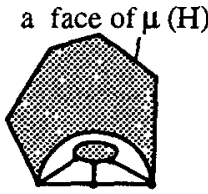

(c)
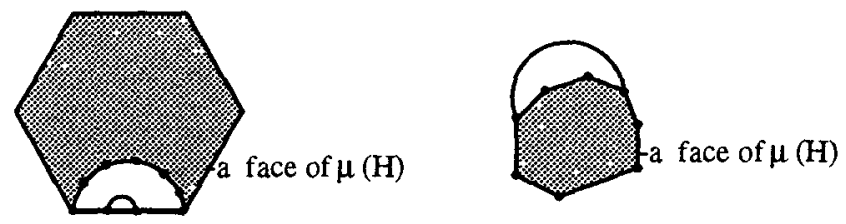

(d)

Fig. 2. Examples of drawings violating conditions (a)-(d).

(d) Any edge e from $E(G)-E(H)$ joining two vertuces of $H$ has endpoints belonging to different straight-lines of $\mu(H)$ and is drawn inside the face containing its endpoints.

Proof: Necessity. Let us prove the necessity of (a). Suppose that $\mu(G)$ is a convex drawing and that there exists a vertex $v$ of $G-O F(G)$ of degree at least three for which (a) is not satisfied (Figure 2 (a)). Then there exists a pair of vertices $u, w$ such that any path joining $v$ to vertices of $O F(G)$ contains $u$ or $w$. Then $\{u, w\}$ divides $G$ into two graphs $G_{1}$ and $G_{2}$ (both containing $u$ and $w$ ), where $G_{1}$ contains $v$. At least one vertex, $z$, of $O F\left(G_{1}\right)$ different from $u$ and $w$ has a strictly convex angle on $O F\left(G_{1}\right)$, because otherwise the drawing of $G_{1}$ would have been a straight-line. (The latter is not possible since the degree of $v$ is $\geq 3$.) Therefore $z$ belongs to a non-convex face of $\mu(G)$ - a contradiction. The necessity of conditions (b), (c), and (d) is established in a similar way (see Figure 2 (b), (c),(d)).

Sufficuency. Assume now that conditions (a)-(d) are satisfied. We will prove by induction on the number of vertices of $G-H$ that $\mu(H)$ can be convexly extended to $\mu(G)$. We consider the following cases.

Case 1: $O F(H) \neq O F(G)$.

There exists a path $p$ of vertices of $O F(G)-O F(H)$ whose endpoints are the endpoints of an edge $e$ of $O F(H)$ (Figure $3(\mathrm{a})$ ). Denote by $F^{*}$ the cycle of $(\dot{x}$ that results after replacing $e$ by $p$ in $O F(H)$. Extend the drawing of $H$ to a strictly convex drawing $\mu\left(H+F^{*}\right)$ of $H+F^{*}$. It is easy to show that the conditions (a)-(d) are satisfied for $\mu\left(H+F^{*}\right)$.

Case 2: $O F(H)=O F(G)$.

Denote by $y$ any vertex of $H$ adjacent to a vertex, say $q$, of $G-H$. Let $Q$ be the face of $\mu(H)$ that contains $q$. By assumption $\mu(Q)$ is a convex polygon. Denote by $p_{1}, p_{2}, \ldots, p_{k}$ the paths corresponding to the sides of $\mu(Q)$. Let $y$ be incident to $p_{1}$ and $p_{k}$ and $y^{\prime}$ and $y^{\prime \prime}$ be the other endpoints of $p_{1}$ and $p_{k}$ respectively (Figure $3(\mathrm{~b}),(\mathrm{c})$ )).

Denote by $G^{\prime}$ the subgraph of $G$ that is drawn inside $Q$ including $Q$. We can assume without loss of generality that $\left({ }^{\prime}\right.$ contains no vertex $z$ of degree 2 because otherwise we can replace $z$ and its two adjacent edges by a single edge. (Note that this replacement will not create parallel edges because of condition (b).) Consider the following two subcases: 


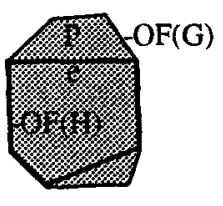

(a)

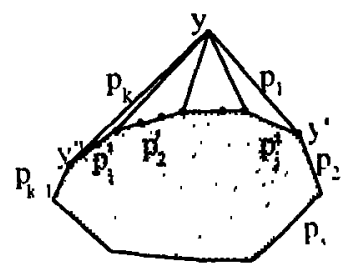

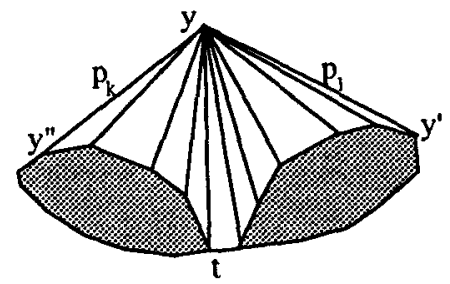

(b)

(c)

Fig. 3. Cases in the sufficiency proof.

Case 2.1: $\left(i^{\prime}-y\right.$ is not biconnected.

There exists a vertex $t$ such that $G-\{y, t\}$ is disconnected. We will show that $t$ belongs to $Q$. For the sake of contradiction assurne that $t$ does not belong to $Q$. Then, according to condition (a), any component of $(i-\{y, t\}$ that contains no vertices from $Q-y$ will have vertices of degree $\leq 2$. This contradicts to the assumption that $G^{\prime \prime}$ contains no vertex of degree 2 . Thus $t$ belongs to $Q$. Moreover, $t$ is not on $p_{1}$ or $p_{k}$ since this will contradict to condition (c) or to the assumption on the minimum degree of $G^{\prime}$. Therefore $t$ belongs to some of the paths $p_{2}, \ldots, p_{k-1}$ and $t \neq y^{\prime}, y^{\prime \prime}$. We can define an edge $(y, t)$ if it is not in $G$ and draw $(y, t)$ as a straight-line segment in $Q$, dividing $Q$ into two convex faces (Figure $3(\mathrm{~b})$ ). The validity of the theorem in this case follows by induction on the number of biconnected components of $G^{\prime}-y$.

Case 2.2.: $G^{\prime}-y$ is biconnected.

Let $Q^{\prime}$ be the face that results when all faces that are incident to $y$ are removed from $Q . Q^{\prime}$ is bounded by the paths $p_{2}, \ldots, p_{k-1}$ and a path $p^{\prime}$ that contains vertices belonging to the same faces as $y$ (Figure $3\left(\mathrm{c}\right.$ )). Partition $p^{\prime}$ into paths $p_{1}^{\prime}, p_{2}^{\prime}, \ldots, p_{j}^{\prime}$ by the neighbors of $y$ as in Figure 3 (c). Draw $p^{\prime}$ convexly inside the triangle $y, y^{\prime}, y^{\prime \prime}$ so that each of the paths $p_{1}^{\prime}, p_{2}^{\prime}, \ldots, p_{j}^{\prime}$ is on a straight-line. Then the cycle $F^{*}$ consisting of $p_{1}^{\prime}, p_{2}^{\prime}, \ldots, p_{j}^{\prime}, p_{2}, \ldots, p_{k-1}$ is convexly drawn. Thus the corresponding extension of the drawing of $\mu(H)$ to drawing, $\mu\left(H+F^{*}\right)$, of $H+F^{*}$ is convex. It is easy to see that conditions (a)-(d) are satisfied for $\mu\left(H+F^{*}\right)$ and, by the inductive assumption, $\mu\left(H+F^{*}\right)$ can be convexly extended to $\mu(G)$.

From Theorem 1 the next statement follows.

Theorem 2 Let $G$ be a planar biconnected embedded graph and $H$ be a biconnected subgraph of $(r$ whose embedding can be extended to a convex drawing of $H$. There exists a convex drawing of $G$ such that the induced drawing of $H$ is convex, if and only if and $G$ and $H$ satisfy the conditions $(a)-(d)$ of Theorem 1 and any vertex of $G$ embedded outside $O F(H)$ can be connected to $O F\left(G^{\prime}\right)$ by a path containing no vertices from $H$. 
Proof (sketch): Consider the embedded graph $G^{\prime}$ resulting. after deletion from $G$ all vertices embedded inside $O F(H)$ excluding the vertices on $O F(H)$. Thus, in $G^{\prime}, H$ corresponds to a single face. If there exists a vertex $v$ of $G$ embedded outside $O F(H)$ such that any path joining $v$ with $O F(C)$ contains a vertex of $H$, then $v$ violates condition (a) of Theorem 1 with respect to the graph $C^{\prime}$. In this case no convex drawing of $C^{\prime}$ and consequently of $G$ exists, which proves the necessity of the conditions of the theorem. If $G$ satisfies the conditions of Theorem 2 then it is easy to check that $G^{\prime}$ satisfies the conditions of Theorem 1 with respect to any strictly convex drawing of $O F(G)$. Consider any convex drawing $\mu\left(G^{\prime}\right)$ of $G^{\prime}$ and extend it to a convex drawing of $G^{\prime}+H$. Now $G^{\prime}$ and the drawing of $C^{\prime}+H$ satisfy the conditions of Theorem 1. Thus there exists a convex drawing of $G$ that induces a convex drawing of $H$.

Another question of interest is whether one can always extend a convex drawing of a subgraph $H$ of $G$, where the drawing of $O F(H)$ is arbitrary. In the full version of the paper we show that this, in general, is not possible and give the conditions which $O F(H)$ should satisfy, in addition to those of Theorem 1, so that the drawing of $H$ is extendible to a convex drawing of $(i$.

We will make use of the following claim which directly follows from Theorems 1 and 2.

Corollary 1 Let $G$ be a planar embedded biconnected graph and $c$ be any chordless cycle of $G$ such that any vertex of $G$ embedded outside $c$ can be connected to $O F(G)$ by a path containing no vertices from $c$. There exists a convex drawing of $G$ such that the induced drawing of $c$ is convex if and only if there exists (any) convex drawing of $G$.

\section{Convex testing}

In this section we investigate the problems of testing if a given planar graph $G$ has a convex drawing in the plane and constructing the drawing if it exists. We use the fact that our proof of Theorem 1 is a constructive one and leads to a linear algorithm for finding a suitable convex extension, if one exists.

Theorem 3 If $G$ and $H$ are graphs satisfying Theorem 1, then a convex extension of $G$ can be found in $O(n)$ time, where $n$ is the number of vertices of $G$.

Proof: Apply to $G$ the algorithm outlined below.

Algorithm EXTEND $(G, H, \mu(H))$

1. If $G$ contains 3 vertices, then the drawing of $G$ is convex. So assume that $G$ contains at least 4 vertices. Determine which case from the proof of Theorem 1 applies (see Figure 3).

If Case 1 applies, find a path $p$ and a cycle $F^{*}$ as in the proof of Theorem 1. Extend the drawing of $O F(H)$ to a strictly convex drawing of $O F\left(H+F^{*}\right)$ and call the procedure $\operatorname{EXTEND}\left(G, H+F^{*}, \mu\left(H+F^{*}\right)\right)$.

If Case 2 applies, pick any vertex $y$ of $H$ adjacent to a vertex $q$ of $G-H$. Let $Q$ be the face of $\mu(H)$ that contains $q$ and $C^{\prime}$ the subgraph of $G$ that is drawn inside $Q$ including $Q$. Denote by $B$ any biconnected component of $G^{\prime}-y$. Construct the path $p^{\prime}$ that contains all vertices of $B$ sharing a face with $y$. Draw $p^{\prime}$ convexly in the triangle determined by $y$ and the endpoints of $p$, as described in the proof of Theorem 1. Call recursively $\operatorname{EXTEND}\left(G, H+p^{\prime}, \mu\left(H+p^{\prime}\right)\right)$. 
The correctness of this algorithm follows from the proof of Theorem 1. The algorithm can be easily implemented in linear time and space as in $[5,12]$.

If we can determine a cycle $c$ of $G$ that can be an outer facial cycle of a convex drawing of $G$, then we can draw $c$ as a strictly convex polygon and then use Algorithm EXTEND described above to extend the drawing of $c$ to a convex drawing of $G$. However, a planar graph $G$ can have an exponential number of cycles that can be faces of some drawing of $C_{i}$ and we have no obvious way of finding an extendible one. Chiba et al. [5] construct a linear algorithm that finds an extendible outer facial cycle of $G$ by applying the algorithms for finding the triconnected components of $G[8]$ and for planarity testing [9] on a graph that contains structural information about $G$ provided by the triconnectivity algorithm. Their algorithm for finding a suitable outer facial cycle, however, is much more complex than the algorithm for drawing the rest of the graph.

Our results presented above give the advantage that one can start with a convex embedding of any cycle of $G$ satisfying the conditions of Corollary 1 , not necessarily a facial one. The consequence is, as we show below, that we can test if a graph has a convex drawing by a conceptually simple algorithm and with a relatively little amount of extra work with respect to the extending algorithm.

Theorem 4 Given any biconnected planar n-vertex graph $G$, one can test if there exists a convex drawing of $G$ and construct such a drawing, if one exists, in $O(n)$ time and space.

Proof (sketch): Assume that a biconnected planar graph $G$ has a convex drawing. (If $G$ has no convex drawing then some of the subsequent steps will fail.) First we will construct a combinatorial embedding of $G$ that corresponds to some convex drawing of $G$ and then we will transform the combinatorial embedding into such a drawing.

Find an initial planar embedding of $G$ by using the linear algorithm of [9]. Let $H$ be a chordless biconnected subgraph of $G$ and let $B$ be any bridge of $G-H$. Assume that there exists an embedding of $B$ in the outer face $h_{\text {out }}$ of $H$ and an embedding of $B$ in an internal face $h_{i n}$ of $H$. We have to choose in which of the two faces to embed $B$. Note that, by our assumption that a convex drawing of $G$ exists, there is always an embedding of $B$ in $h_{\text {out }}$ that is convexly extendible to $B+H$ (by Corollary 1 ), and the same embedding of $B$ (as well as any embedding of $B$, ) with $B$ moved in $h_{i n}$, is convexly extendible to $B+H$ (by conditions (a) $-(\mathrm{d})$ of Theorem 1).

This observation motivates the following algorithm for updating the embedding of G.

\section{Algorithm UPDATE (H)}

1. Initially let $H$ be the empty graph. Construct a path $p$ containing at least one vertex of $C-H$ and such that $H+p$ is outer-chordless and biconnected. Let $H:=H+p$. Repeat the following steps for any bridge $B$ of $C-H$.

2 . If there exists an embedding of $B$ in $h_{\text {out }}$, then recursively find a convexly extendible embedding of $B$ in $h_{\text {out }}$. During this step, determine if some vertices of $B$ have to be placed on the outer face of $G$ (call such vertices anchored) in order to satisfy conditions (a)-(c) of Theorem 1.

3. If $B$ contains exactly two attachment edges and either: (i) $B$ contains a vertex not in $H$ of degree three or more, or (ii) $B$ and some other bridge of $G-H$ are both simple paths sharing the same endpoints and $B$ is not a single edge, or (iii) $B$ is marked as containing an anchored vertex, then mark $B+H$ as containing an anchored vertex too. 
4. If $B$ contains anchored vertices and $B$ is embedded in $h_{i n}$, then move $B$ into $h_{\text {out }}$, possibly forcing other bridges to be moved in order to preserve the planarity of the current embedding of $G$.

It is easy to show that the total time needed for moving all bridges by Algorithm EXTEND is proportional to the number of edges of $G$ which is $O(n)$. Thus the only nontrivial step in the linear implementation of Algorithm EXTEND is the fast construction of the path $p$ in Step 1, which can be done in time $O(|p|)$ plus time that, for all iterations of the algorithm, adds up to $O(n)$. For the efficient implementation of this step we preprocess $G$ by finding an st-numbering [7] of its vertices and defining a spanning tree $T$ of $G$, where the parent of each vertex $v \neq s$ of $G$ is the vertex adjacent to $v$ with the lowest $s t$-number. Since any path in $T$ from a vertex to the root of the tree is chordless, it is easy to show that all paths $p$ can be constructed in the desired time bounds.

Finally, we obtain a convex drawing of $(r$ corresponding to the embedding found by Algorithm UPDATE by applying Algorithm EXTEND with $H:=O F(G)$ and choosing $\mu(H)$ to be any strictly convex drawing of $O F(G)$.

\section{References}

1. G. Di Battista, P. Eades, R. Tamassia, and I.G. Tollis, Algorithms for drawing graphs: an annotated bibliography, Technical Report, Brown University, 1988; updated 1993.

2. Guiseppe Di Battista, Roberto Tamassia, and Luca Vismara, On-line convex planarty testing, Proc. WG'94, in Lecture Notes in Computer Science, Springer-Verlag, to appear.

3. K. Booth, G. Lueker, Testing for the consecutive ones property, interval graphs, and graph planarity using PQ-tree algorithm, J. Comp. Syst. Sci. 13, 1976, pp. 335-379.

4. N. Chiba, T. Nishizeki, S. A be, T. Ozawa, $A$ linear algorithm for embedding planar graphs using PQ-trees, J. Comput. System Sci. 30, 1985, 54-76.

5. N. Chiba, T. Yamanouchi, T. Nishizeki, Linear algorithms for convex drawings of planar graphs, in Progress in Graph Theory, J.A. Bondy and U.S.R. Murty (eds.), Academic Press, 1984, pp. 153-173.

6. M. Chrobak and (i. Kant, Convex grid drawengs of 3-connected planar graphs, Technical Report RUU-CS-93-45, Utrecht University, 1993.

7. S. Even and R.E. Tarjan, Computıng an st-numbering, Theor. Comput. Sci. 2, 1976, 339344 .

8. J. Hopcroft and R.E. Tarjan, Duvtding a graph into triconnected components, SLAM J. Comput. 2, 1973, pp. 135-158.

9. J. Hopcroft and R.E. Tarjan, Efficient planarty testing, J.ACM, 21:4, 1974, pp. 549-568.

10. G. Kant, Drawng planar graphs using the lmc-ordering, Proc. IEEE Symp. on Foundations of Computer Science, 1992, pp. 101-110.

11. A. Lempel, S. Even, I. Cederbaum, An algorithm for planarity testing of a graph, Theory of Graphs: International Symposium, (Gordon and Breach, New York, 1967, pp. 215-232.

12. T. Nishizeki, N. Chiba, Planar Graphs: Theory and Algorithms, North Holland, 1988.

13. W. Schnyder and W. Trotter, Convex drawings of planar graphs, Abstracts of the AMS, vol. 13, 1992.

14. S.K. Stein, Convex maps, Proc. Amer. Math. Soc., vol. 2, pp. 464-466, 1951.

15. C. Thomassen, Planarity and duality of finite and infinite planar graphs, J. Combinatorial Theory, Series B 29, 1980, pp. 244-271.

16. W.T. Tutte, Convex representations of graphs, Proc. London Math Soc., vol. 10, 1960, pp. 304-320.

17. W.T. Tutte, How to draw a graph, Proc. London Math Soc., vol. 3, no. 13, 1963, pp. 743-768.

18. K. Wagner, Bemerkungen zum Vierfarbenproblem, Jber. Deutsch. Math.-Verein, vol. 46, 1936, pp. 26-32. 